\title{
No-Risk - Um Método para Aplicação de Gerência de Riscos em Projetos de Software
}

\author{
Gustavo C. Oliveira ${ }^{1}$, Ricardo M. Bastos ${ }^{1}$ \\ ${ }^{1}$ Pontifícia Universidade Católica do Rio Grande do Sul - PUCRS \\ Programa de Pós-Graduação em Ciência da Computação \\ Porto Alegre - RS - Brasil \\ \{goliveira, bastos\} @inf.pucrs.br
}

\begin{abstract}
This paper shows a method of software project risk management, called No-Risk, conceived from a developed preliminary study in the form of a searches applied the project managers. Such study had as objective to identify which they are the relevant risk factors in projects of this nature, associates to the cost, schedule and quality dimensions. This method was elaborated for integration with the software development process of a information technology company.
\end{abstract}

Resumo. Este artigo apresenta um método para gerência de riscos em projetos de software, denominado No-Risk, concebido a partir de um estudo preliminar desenvolvido na forma de uma pesquisa junto a gerentes de projeto. Tal estudo teve como objetivo identificar quais são os fatores de risco relevantes em projetos desta natureza, associados às dimensões de custo, prazo e qualidade. Este método foi elaborado para integração com o processo de desenvolvimento de software de uma empresa de tecnologia da informação.

\section{Introdução}

De acordo com o Standish Group (2004), em 2002, um estudo desenvolvido, denominado "Chaos", relatou que $66 \%$ dos projetos de software apresentavam erros de previsão de cronograma, orçamento e/ou qualidade; destes, $15 \%$ dos projetos foram cancelados. Já em 2004, a pesquisa apresentou um aumento no índice de falhas de projetos de software obtendo um percentual de $18 \%$.

A Tabela 1 apresenta o resultado das pesquisas realizadas pelo Standish Group nos últimos 10 anos relacionada ao percentual de:

- Sucesso: projeto concluído dentro do prazo e orçamento previstos, com todas as características e funções, conforme especificado inicialmente;

- Falha: projeto cancelado em algum ponto durante o ciclo de desenvolvimento;

- Replanejamento: projeto concluído, porém acima do orçamento e em atraso, ou ainda incompleto em relação às características e funções especificadas originalmente.

Tabela 1. Estudos do Standish Group (1994 a 2004)

\begin{tabular}{c|c|c|c|c|c|c}
\hline Ocorrência & $\mathbf{1 9 9 4}(\boldsymbol{\%})$ & $\mathbf{1 9 9 6}(\boldsymbol{\%})$ & $\mathbf{1 9 9 8}(\boldsymbol{\%})$ & $\mathbf{2 0 0 0}(\boldsymbol{\%})$ & $\mathbf{2 0 0 2}(\boldsymbol{\%})$ & $\mathbf{2 0 0 4}(\mathbf{\%})$ \\
\hline Sucesso & 16 & 27 & 26 & 28 & 34 & 29 \\
\hline Falha & 31 & 40 & 28 & 23 & 15 & 18 \\
\hline Replanejamento & 53 & 33 & 46 & 49 & 51 & 53 \\
\hline
\end{tabular}


Percebe-se nesta pesquisa a existência de um elevado índice de insucesso em projetos de software. A partir desta constatação, surge a necessidade da identificação dos fatores de risco de um projeto de software. O correto dimensionamento destes fatores permitiria um melhor tratamento dos possíveis riscos inerentes à execução do projeto, sendo, portanto essencial para a adequação de um método de Gerência de Riscos em Projetos de Software (GRPS).

O presente trabalho tem como objetivo apresentar o No-Risk. Trata-se de um método para GRPS desenvolvido para integração com o processo de desenvolvimento de software da empresa CWI Software Ltda. A CWI foi fundada em 1991, em Porto Alegre - RS, e hoje atua na área de tecnologia da informação distribuindo seus serviços entre duas unidades de negócio e uma unidade técnica, sendo esta última localizada no Pólo de Informática de São Leopoldo - RS.

Para a definição do No-Risk foram utilizadas como referência propostas de métodos apresentados na literatura e uma pesquisa realizada em nível nacional para identificação dos principais fatores de risco em projetos de software. A principal contribuição deste trabalho está na definição de um método para GRPS associado a um processo de desenvolvimento de software, cuja proposta tem como premissa básica a análise de riscos baseada em fatores de risco previamente mapeados.

A seção 2 apresenta a pesquisa realizada junto a gerentes de projeto de software com o objetivo de mapear e classificar riscos em projetos de software. A seção 3 apresenta o método proposto, desenvolvido com base na pesquisa apresentada na seção 2 e em trabalhos similares na literatura. Na seção 4 são realizadas considerações finais e discutidos trabalhos futuros.

\section{Levantamento de Impacto de Riscos na Percepção de Gerentes de Projeto}

Durante o ano de 2005, realizou-se uma pesquisa junto a gerentes de projetos, na forma de um questionário, com o objetivo de identificar e quantificar os principais fatores de risco em projetos de software para sistemas de informação. O questionário compreendeu perguntas de múltipla escolha na forma de ordenação das respostas de acordo com a sua prioridade. No total, foram apresentados 29 fatores de risco definidos com base em Sommerville (2003), Wallace e Keil (2004) e entrevistas realizadas com pesquisadores da área.

Participaram da pesquisa gerentes de projeto vinculados a empresas de Porto Alegre da área de Tecnologia da Informação, bem como profissionais da área de todo território brasileiro cadastrados em grupos de discussão relacionados ao PMI. A amostra de respondentes apresentou caráter não-probabilístico espontânea, pois foi aplicada a gerentes de projetos que se prontificaram a responder à pesquisa. Coletou-se ao todo 61 questionários válidos do total de convidados para o seu preenchimento. O percentual de participação dos respondentes por regiões do Brasil é apresentado conforme Tabela 2. 
Tabela 2. Distribuição dos Respondentes por região

\begin{tabular}{c|c|c}
\hline Região & Respondentes & $\%$ \\
\hline Centro-Oeste & 5 & 8,20 \\
DF & 11 & 18,03 \\
Nordeste & 6 & 9,84 \\
Norte & 0 & 0,00 \\
Sudeste & 20 & 32,79 \\
Sul & 19 & 31,15 \\
\hline Total & 61 & 100 \\
\hline
\end{tabular}

A caracterização da amostra na qual foi aplicada esta pesquisa é apresentada na Tabela 3.

Tabela 3. Caracterização da Amostra

\begin{tabular}{l|c|c|c}
\hline Item & Intervalo & Média & Desvio Padrão \\
\hline Faixa etária & 21 a 51 anos & 32,33 anos & $\pm 6,59$ \\
\hline Experiência professional & 1 a 31 anos & 10,49 anos & $\pm 6,10$ \\
\hline Experiência como gerente de projetos & 1 a 15 anos & 4,65 anos & $\pm 3,29$ \\
\hline Tempo médio de duração dos projetos & 1 a 26 meses & 8,73 meses & $\pm 5,38$ \\
\hline Tamanho médio das equipes de projeto & 3 a 20 pessoas & 7,67 pessoas & $\pm 4,87$ \\
\hline Projetos concluídos em 2004 & 0 a 50 projetos & 4,43 projetos & $\pm 3,05$ \\
\hline Projetos em andamento em 2005 & 0 a 20 projetos & 7,13 projetos & $\pm 3,04$ \\
\hline
\end{tabular}

A maioria das empresas nas quais os respondentes atua possuem acima de 100 funcionários, apresentando um percentual de 78,7\% do total de 61 respondentes.

As dimensões de projeto foram classificadas de acordo com as respostas apresentadas em relação às suas ordens indicadas para cada fator de risco, ou seja, foram calculadas as médias das respostas de prioridade alta $($ valor $=3$ ), média (valor $=$ 2) e baixa (valor $=1$ ) apresentadas nas dimensões de projeto obtidas em cada fator de risco apresentado no questionário, conforme Figura 1.

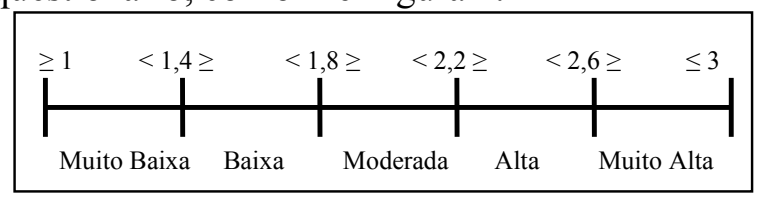

Figura 1. Classificação de Impacto dos Fatores de Riscos de Projetos de Software.

A dimensão prazo foi considerada pela maioria dos respondentes como a mais crítica em projetos de software, apresentando $42,67 \%$ das respostas como prioridade Alta, independente do fator de risco. Já com relação ao custo, percebe-se que não existe uma grande preocupação em relação a esta dimensão, visto que 44,26 \% dos respondentes a definiram como de prioridade Baixa, independente do fator de risco.

Nenhum fator de risco apresentado no questionário se enquadrou na classificação de prioridade Muito Baixa. Já com relação à prioridade Muito Alta, o fator de risco "Doença de pessoas chave da equipe" foi o único enquadrado nesta classificação, no que se refere ao impacto no prazo de projeto. Alguns fatores importantes referentes à prazo de projetos, identificados como prioridade Alta, foram:

- Desnível de treinamento do pessoal em relação à função realizada;

- Dificuldade de recrutamento de pessoal para a montagem da equipe do projeto; 
- Contínuas mudanças de requisitos ou adição de mais funcionalidades/características do que o necessário;

- Influências do ambiente externo no projeto (ex.: mudanças na legislação afetando os requisitos do software);

- Falta de poder de negociação do gerente de projetos junto ao cliente;

- Informações pré-existentes ignoradas;

- Indisponibilidade de recursos de hardware no momento da execução do projeto;

- Utilização de nova tecnologia no projeto (hardware e/ou software).

Logo, concluiu-se que a dimensão prazo foi considerada para esta amostra da população como a mais crítica à GRPS, obtendo uma média final de prioridade de 2,26. Para esta dimensão, os fatores que devem ser vistos com maior atenção no método proposto são aqueles que apresentaram nível de impacto Alto ou Muito Alto, totalizando 19 fatores de risco.

\section{Método Proposto}

Conforme apresentado na introdução deste artigo, este trabalho tem como propósito definir um GRPS integrado ao processo de gerenciamento de projetos adotado pela empresa CWI. Neste sentido, inicialmente foram analisados métodos de GRPS disponíveis na literatura, destacando-se:

- Riskit: assemelha-se à estrutura proposta pelo paradigma do SEI (2004), desprezando apenas a etapa de comunicação de riscos [Kontio 1996];

- Odyssey: utiliza uma infra-estrutura de desenvolvimento de software orientada ao reuso baseada no desenvolvimento de modelos e de componentes reutilizáveis, através de um processo de engenharia de domínio [Braga et al 1999];

- A-Risk: possui foco na identificação e quantificação de riscos de prazo de projeto, que pode ser aplicado antes e durante o desenvolvimento do mesmo, ou seja, em todas as suas fases [Machado 2002].

A partir destes métodos, conclui-se que o método Riskit é o mais abrangente em relação aos demais apresentados, adotando-se como referência para a elaboração desta proposta, pois, de todas as etapas de GRPS previstas na literatura estudada, apenas a etapa de comunicação de riscos, destacada pelo SEI (2004), não é contemplada.

Esta seção apresenta a descrição do método No-Risk, onde é proposta uma forma de gerenciar riscos de projetos de software para sistemas de informação, focando os fatores de risco evidenciados como de prioridade alta ou muito alta na pesquisa apresentada na seção 2. Também será apresentada a integração do No-Risk com o processo de desenvolvimento de projetos de software da CWI.

\subsection{Etapas do No-Risk}

$\mathrm{Na}$ Tabela 4 é apresentada cada uma das etapas do No-Risk, sendo identificados os procedimentos herdados do Riskit, e as extensões propostas para o No-Risk, visando sua integração com o processo da CWI. Para melhor descrever as etapas do No-Risk, são utilizados Diagramas de Atividades da UML apresentados nas Figuras 2 a 7. 
Tabela 4. Etapas e Atividades No-Risk

\begin{tabular}{|c|c|c|}
\hline Etapas No-Risk & Procedimentos Riskit & Extensões No-Risk \\
\hline $\begin{array}{l}\text { Planejamento da Gerência de Riscos: } \\
\text { envolve a identificação específica do } \\
\text { escopo do projeto no contexto da } \\
\text { gerência de riscos, conforme Figura } 2 \text {. }\end{array}$ & $\begin{array}{l}\text { Define escopo e } \\
\text { freqüência da GRPS. } \\
\text { Todos stakeholders são } \\
\text { reconhecidos. } \\
\text { Objetivos indicados no } \\
\text { projeto são revistos e } \\
\text { refinados. }\end{array}$ & $\begin{array}{l}\text { Agrega questões relevantes propostas } \\
\text { pelo MSF (2004), a fim de documentar o } \\
\text { plano de execução do processo da } \\
\text { gerência de riscos. }\end{array}$ \\
\hline $\begin{array}{l}\text { Identificação de Riscos: identifica os } \\
\text { riscos potenciais do projeto de software, } \\
\text { conforme Figura } 3 \text {. }\end{array}$ & $\begin{array}{l}\text { Identifica um grande } \\
\text { número de possíveis } \\
\text { ameaças ao projeto. } \\
\end{array}$ & $\begin{array}{l}\text { Agrega modelo de identificação de riscos } \\
\text { de [Machado 2002]. }\end{array}$ \\
\hline $\begin{array}{l}\text { Análise de Riscos: analisa os fatores } \\
\text { identificados na etapa anterior } \\
\text { qualitativamente, priorizando os efeitos } \\
\text { dos riscos nos objetivos do projeto } \\
\text { através de uma descrição do seu impacto, } \\
\text { e quantitativamente, determinando a } \\
\text { probabilidade e impacto dos riscos e } \\
\text { estimando suas implicações nos objetivos } \\
\text { do projeto classificadas a partir de } \\
\text { atributos numéricos, conforme Figura } 4 .\end{array}$ & $\begin{array}{l}\text { Unifica riscos por } \\
\text { similaridades. } \\
\text { Desenvolve Cenários de } \\
\text { Risco. } \\
\text { Prioriza cenários de risco } \\
\text { baseado nas estimativas } \\
\text { de probabilidade e perda } \\
\text { de utilidade para cada } \\
\text { cenário. }\end{array}$ & $\begin{array}{l}\text { Agrega às três atividades do Riskit a } \\
\text { utilização de níveis de impacto dos } \\
\text { fatores de risco em projetos para a } \\
\text { definição de prioridades. Para a } \\
\text { determinação dos fatores de risco é } \\
\text { utilizada como referência a classificação } \\
\text { obtida para cada fator de risco na pesquisa } \\
\text { apresentada na Seção } 2 \text {. } \\
\text { Define escalas de probabilidades de } \\
\text { ocorrência conforme [Machado 2002]. } \\
\text { Conceito de perda de utilidade e tratado } \\
\text { como nível de exposição ao risco } \\
\text { [Machado 2002]. }\end{array}$ \\
\hline $\begin{array}{l}\text { Planejamento de Controle de Riscos: } \\
\text { elabora o plano de ação de risco e seu } \\
\text { relatório de situação associado. Para cada } \\
\text { fator de risco identificado como } \\
\text { prioridade alta ou muito alta para a } \\
\text { dimensão de prazo de projetos na análise } \\
\text { dos resultados do questionário } \\
\text { apresentado na seção anterior, adotar } \\
\text { estratégias/ações a serem tomadas } \\
\text { [Sommerville 2003] [Jalote 2002], } \\
\text { conforme Figura 5. }\end{array}$ & $\begin{array}{l}\text { Riscos mais importantes } \\
\text { são selecionados para a } \\
\text { definição de ações. } \\
\text { Ações são } \\
\text { implementadas. }\end{array}$ & $\begin{array}{l}\text { Apresenta regras de redução do nível de } \\
\text { exposição ao risco dos cenários do MSF } \\
\text { (2004) com foco no prazo de projetos, } \\
\text { além de alternativas para a elaboração do } \\
\text { plano, como: pesquisa, aceitação, } \\
\text { prevenção, transferência, mitigação e } \\
\text { contingência. }\end{array}$ \\
\hline $\begin{array}{l}\text { Monitoração de Riscos: atualiza o plano } \\
\text { de riscos em função de novas percepções } \\
\text { referentes aos riscos no projeto, conforme } \\
\text { Figura } 6 \text {. }\end{array}$ & $\begin{array}{l}\text { Situação dos riscos é } \\
\text { monitorada em função } \\
\text { das métricas definidas } \\
\text { para cada ação. }\end{array}$ & $\begin{array}{l}\text { Procura observar se algum risco tornou-se } \\
\text { problema, além de identificar novos } \\
\text { riscos e ações, e mudanças de exposição } \\
\text { ao risco atribuídas a um determinado } \\
\text { cenário de risco [PMBOK 2000]. }\end{array}$ \\
\hline $\begin{array}{l}\text { Comunicação de Riscos: mantém os } \\
\text { stakeholders informados sobre qualquer } \\
\text { evento ocorrido durante o andamento do } \\
\text { plano de gerência de riscos, conforme } \\
\text { Figura } 7 \text {. }\end{array}$ & & $\begin{array}{l}\text { Comunica stakeholders envolvidos no } \\
\text { projeto sobre andamento do plano de } \\
\text { gerência de riscos [SEI 2004]. }\end{array}$ \\
\hline $\begin{array}{l}\text { Aprendizagem de Riscos: focaliza no } \\
\text { armazenamento das informações do plano } \\
\text { de GRPS em uma base de conhecimento, } \\
\text { com o objetivo de registrar as lições } \\
\text { aprendidas. }\end{array}$ & $\begin{array}{l}\text { Realiza a atividade de } \\
\text { aprendizagem através do } \\
\text { uso do formalismo } \\
\text { gráfico de riscos. }\end{array}$ & $\begin{array}{l}\text { Permite a navegação entre os riscos } \\
\text { similares, projetando enfrentar os mesmos } \\
\text { riscos apresentados em projetos anteriores } \\
\text { [Braga et al 1999]. }\end{array}$ \\
\hline
\end{tabular}




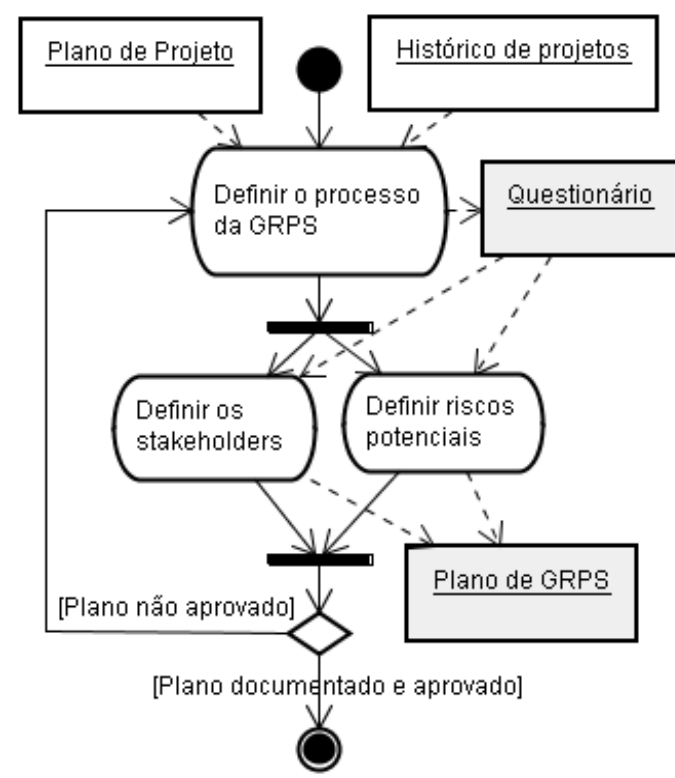

Figura 2. Planejamento da Gerência de Riscos.

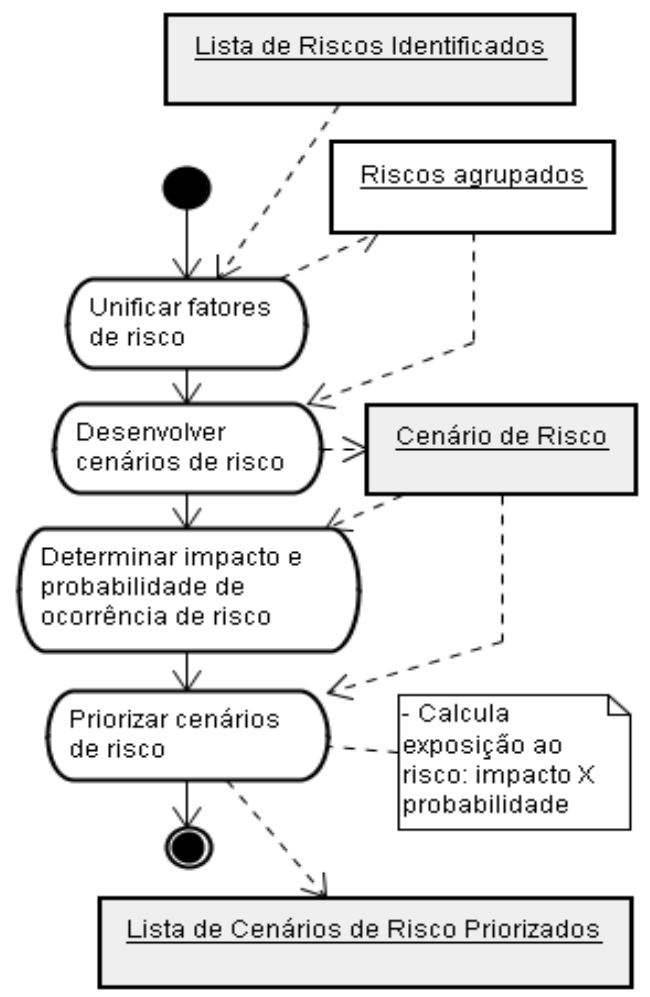

Figura 4. Análise de Riscos.

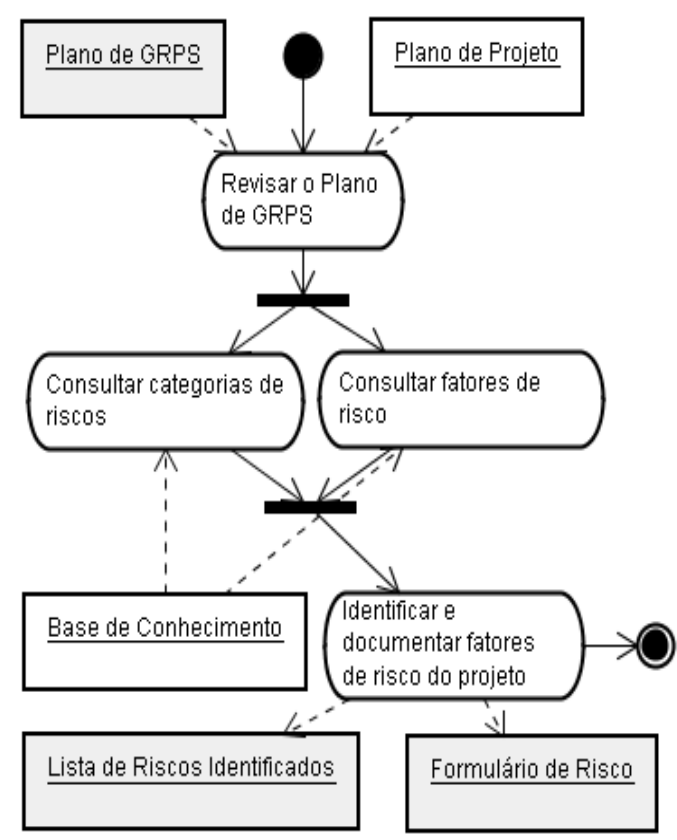

Figura 3. Identificação de Riscos.

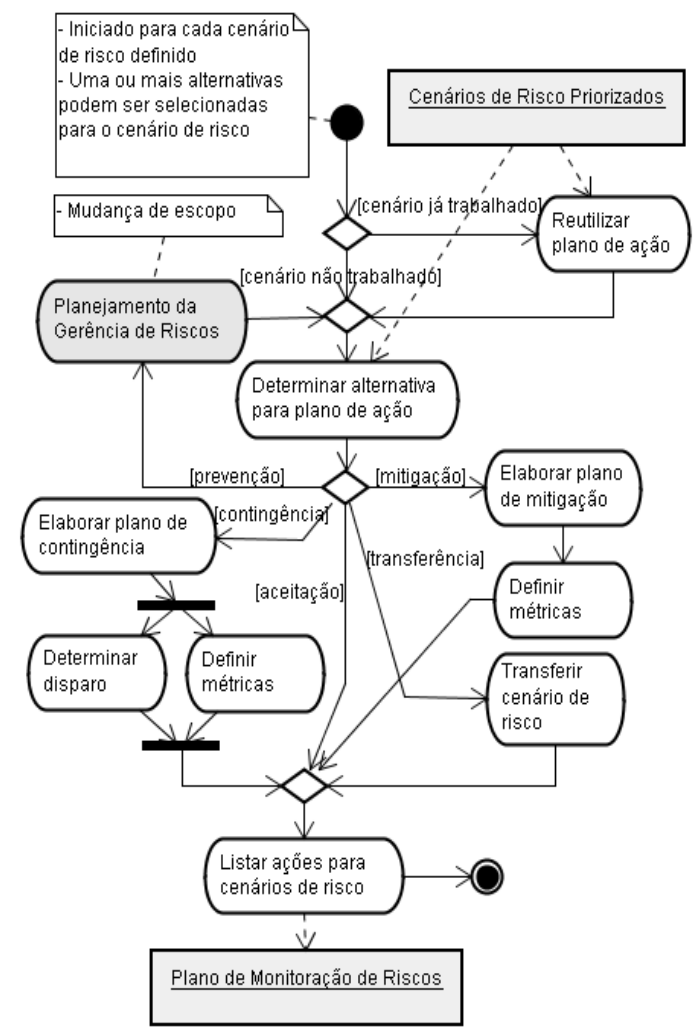

Figura 5. Planejamento do Controle de Riscos. 


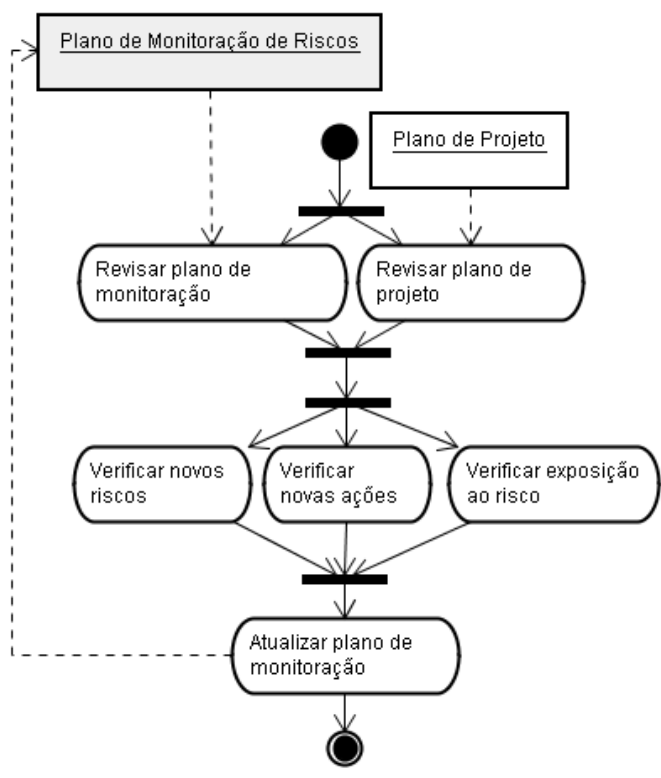

Figura 6. Monitoração de Riscos.

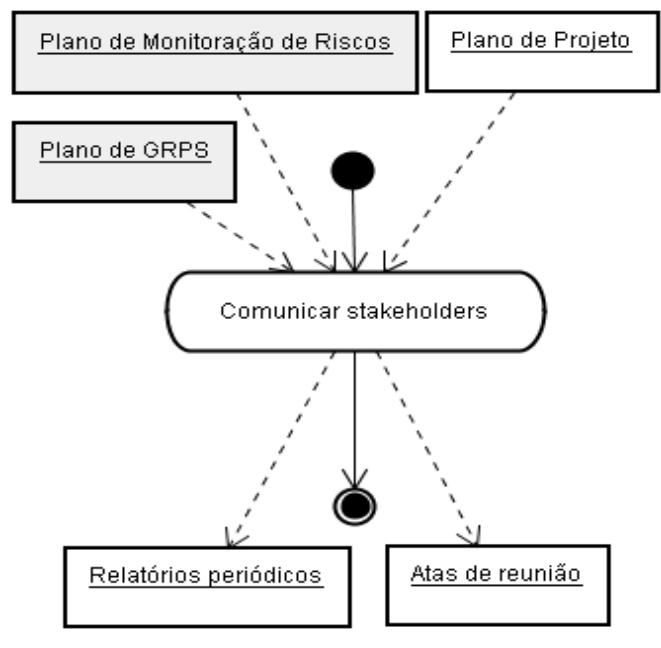

Figura 7. Comunicação de Riscos.

\subsection{Responsabilidades no No-Risk}

Os papéis envolvidos e suas responsabilidades em cada etapa do No-Risk foram definidas baseadas no PMBOK (2000), conforme segue:

- Gestor de Projetos (GP): profissional cedido pelo cliente;

- Usuário Líder (UL): representa todos os usuários do sistema;

- Coordenador de Projetos (CP): responsável pelo gerenciamento do projeto;

- Analista Líder (AL): possui alocação integral ao projeto (100\%) e exerce, além das funções de analista de sistemas.

A Figura 8 apresenta um Diagrama de Casos de Uso identificando cada uma das etapas do No-Risk como sendo um caso de uso, e os atores representando os papéis acima identificados.

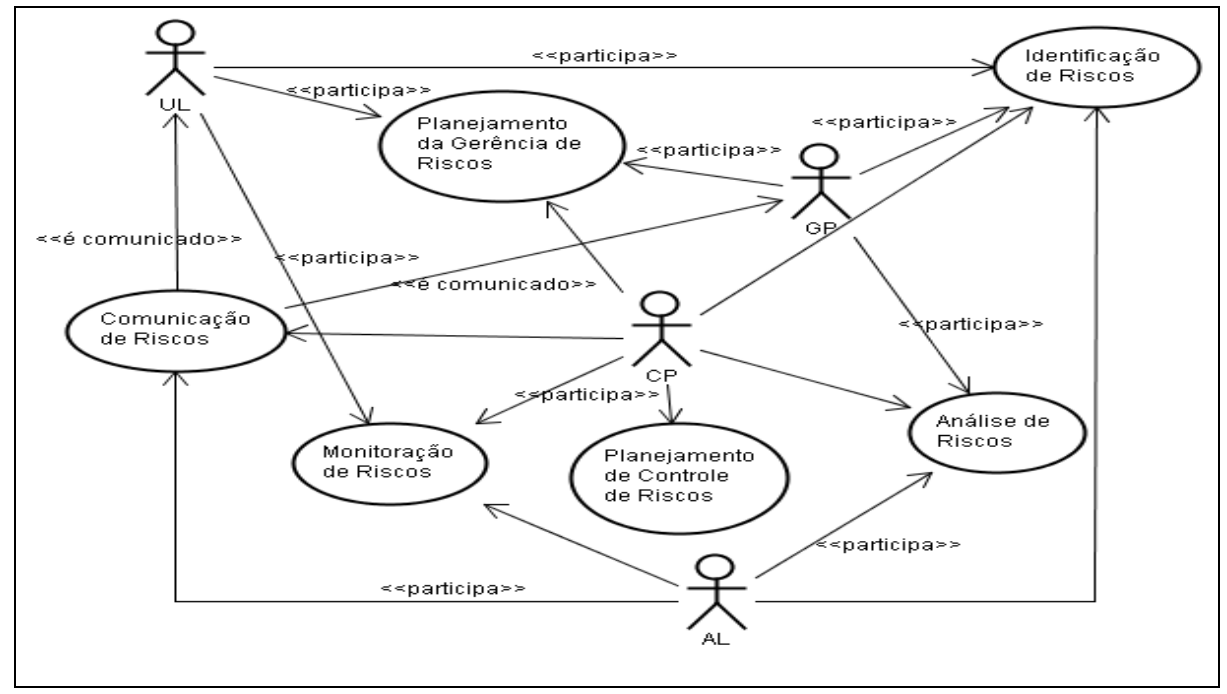

Figura 8. Responsabilidades no No-Risk. 


\subsection{O Processo de Desenvolvimento de Software da CWI e o No-Risk}

O processo aplicado pela CWI (Figura 9) contempla todas as fases do desenvolvimento de um sistema, do levantamento dos objetivos à implantação, o que possibilita a gestão completa do processo.

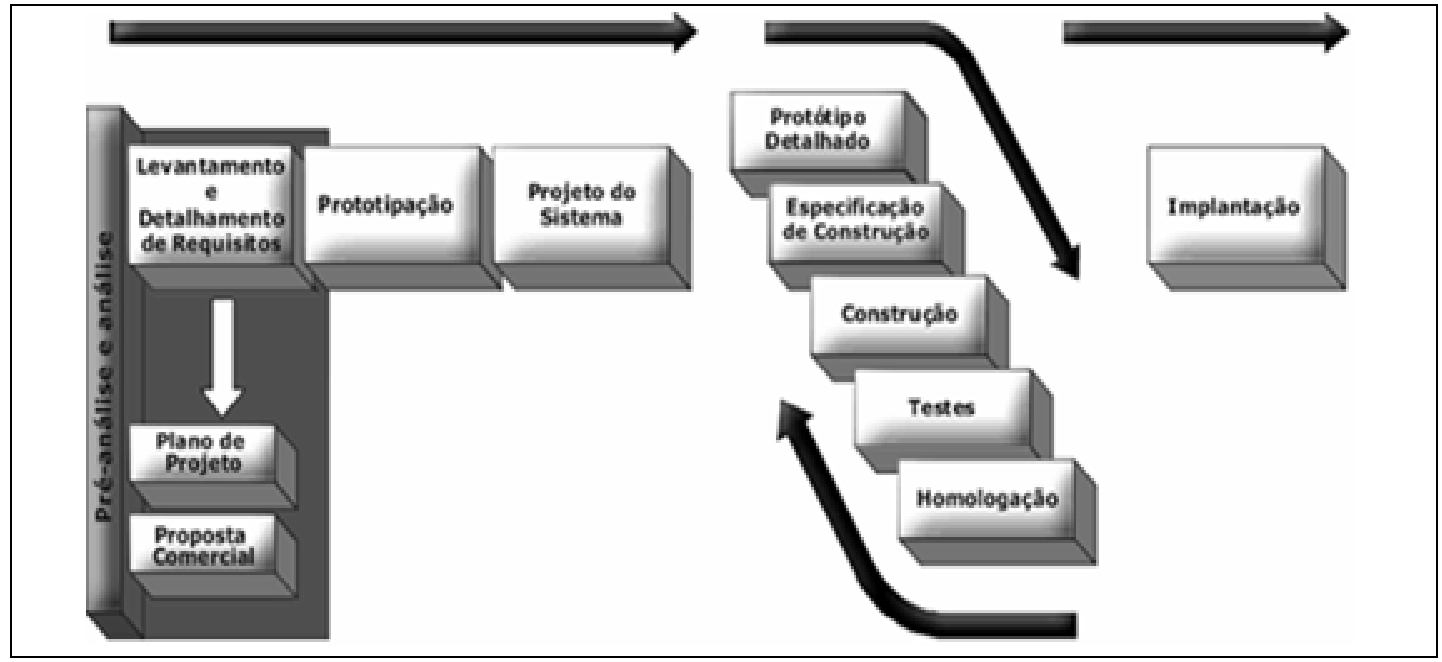

Figura 9. Etapas de Desenvolvimento na CWI.

A Tabela 5 apresenta a relação entre as principais atividades realizadas no processo CWI com as etapas propostas pelo método No-Risk. A GRPS se inicia apartir do momento em que a Proposta Comercial (Figura 9) do software a ser desenvolvido é aprovada. Salienta-se que as etapas do No-Risk não ocorrem em paralelo, ou seja, na atividade "Protótipo Detalhado", por exemplo, as etapas em destaque ocorrem sequencialmente.

Tabela 5. Relação entre as atividades CWI e o método No-Risk.

\begin{tabular}{|c|c|c|c|c|c|c|c|c|c|}
\hline \multirow[b]{2}{*}{ Etapas do Método No-Risk } & \multicolumn{9}{|c|}{$\begin{array}{l}\text { Metodologia de Desenvolvimento CWI } \\
\text { baseada no PMBOK }\end{array}$} \\
\hline & 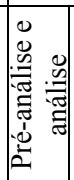 & 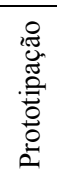 & 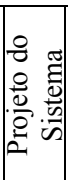 & 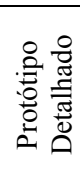 & 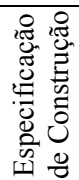 & 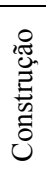 & $\stackrel{\mathscr{\varpi}}{\tilde{D}}$ & 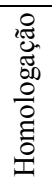 & 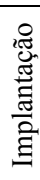 \\
\hline $\begin{array}{l}\text { Planejamento da Gerência de } \\
\text { Risco }\end{array}$ & & & & & & & & & \\
\hline Identificação de Riscos & & & & & & & & & \\
\hline Análise de Riscos & & & & & & & & & \\
\hline $\begin{array}{l}\text { Planejamento de Controle de } \\
\text { Riscos }\end{array}$ & & & & & & & & & \\
\hline Monitoração de Riscos & & & & & & & & & \\
\hline Comunicação de Riscos & & & & & & & & & \\
\hline Aprendizagem de Riscos & & & & & & & & & \\
\hline
\end{tabular}

\section{Considerações Finais e Trabalhos Futuros}

O presente trabalho teve como foco a definição de um método para GRPS para projetos de software. O principal diferencial em relação aos métodos utilizados como referência (vide Seção 3), está no fato de sua construção ter sido realizada a partir de um estudo 
preliminar desenvolvido na forma de uma pesquisa junto a gerentes de projeto. Tal estudo teve como objetivo identificar quais são os fatores de risco relevantes em projetos desta natureza, associados às dimensões de custo, prazo e qualidade. Os resultados da pesquisa permitiram a definição de um modelo probabilístico proposto para a realização da análise de riscos no método proposto (etapa Análise de Riscos da Tabela 4). O No-Risk foi desenvolvido para integração com o processo de desenvolvimento de software da empresa de tecnologia da informação CWI Software.

No momento, o No-Risk está sendo aplicado em projetos de software na CWI, cujos resultados de seu uso estão sendo registrados para análise ao final dos projetos. Também está sendo desenvolvida uma ferramenta de software para apoio ao uso do NoRisk, onde se pretende registrar as informações associadas à GRPS em um banco de conhecimento. O uso destas informações fornecerá assistência ao gerenciamento do risco por toda a organização, permitindo sua disseminação e compartilhamento por todos os participantes de projetos de software da empresa.

\section{Referências}

Braga, R. Werner, C. Mattoso, M. (1999), "Odyssey: A Reuse Enviroment Based on Domain Models", 2nd IEEE Symposium on Application Specific Systems and Software Engineering Technology, ASSET’99, Richardson, USA, March.

Jalote, P. (2002), "CMM in practice: process for executing software projects at Infosys", SEI series in software engineering, ISBN, p. 159-174, May.

Kontio, J. (1996), “The Riskit Method for Software Risk Management. Version 1.00", Computer Science Technical Reports, University of Maryland. College Park, MD.

Machado, C. A. (2002), “A-RISK: Um Método para Identificar e Quantificar Risco de Prazo em Projetos de Desenvolvimento de Software", Dissertação de Mestrado. Pontifícia Universidade Católica do Paraná - PUCPR. Curitiba.

MICROSOFT SOLUTIONS FRAMEWORK. (2004), MSF - Risk Management Process, http://www.microsoft.com/msf, October.

PMBOK Guide. (2000), Um Guia do Conjunto de Conhecimentos do Gerenciamento de Projetos. Newtown Square, PA, USA: Project Management Institute.

SEI. (2004), Software Performance Institute. Pittsburgh, http://www.sei.cmu.edu, October.

Sommerville, I. (2003), Engenharia de Software, São Paulo, Ed. Addison Wesley.

THE STANDISH GROUP. (2004), Chaos Report. http://www.standishgroup.com, October.

Wallace, L. Keil, M. (2004), "Software Project Risks And Their Effect On Outcomes", Communications of The ACM, vol. 47, nr. 4, p. 68-73, April. 